\title{
50 años de Relaciones Públicas en España. De la propaganda y la publicidad a la gestión de la reputación
}

\section{Years of Public Relations in Spain. From Propaganda and Advertising to Reputation Management}

\section{Elena Gutiérrez García. Universidad de Navarra}

Natalia Rodríguez Salcedo. Universidad de Navarra

Recibido: 29-I-2009 - Aceptado: 29-VI-2009

Resumen:

Desde que hace cincuenta años apareciera en España el término 'relaciones públicas' en un libro académico, profesión y disciplina no han hecho más que crecer, aunque continúa el debate sobre su propia identidad. La profesión comenzó en un régimen dictatorial, pero ha sido en las últimas tres décadas cuando se ha observado un crecimiento exponencial. El artículo realiza un análisis histórico del último medio siglo, fundamentado en fuentes secundarias y primarias -publicaciones académicas primigenias, entrevistas a los pioneros y documentación inédita del archivo de la primera agencia de relaciones públicas, la Sociedad Anónima Española de RP, fundada en 1960.

Palabras clave:

Relaciones públicas, España, historia, profesionalización

Abstract:

It has been fifty years since the term 'public relations' first appeared in a Spanish academic textbook. Ever since, the profession and the academic fields have continued growing in importance. However, there is an ongoing discussion about the nature of PR. While the profession developed in a non-democratic regime in Spain, it experienced exponential growth with democracy. This paper analyses its historical development during the last half of the 20 th century through use of primary and secondary sources -communication journals, oral interviews with the pioneers, and documentary research into the archive of the first Spanish PR agency established in 1960.

Key Words:

Public Relations, Spain, History, Professionalization 


\section{Introducción}

Las relaciones públicas ocupan un modesto lugar en los estudios de la comunicación en España. Los primeros escritos teóricos sobre relaciones públicas y las primeras campañas no aparecieron en nuestro país hasta la década de los años 50 del siglo XX. Quizás por esta razón apenas existen algunos apuntes breves recientes sobre la profesión y su desarrollo histórico (Arceo, 2004a, 2004b; Arceo, 2006; Checa, 2007; Moreno, 2004; Noguero i Grau, 2004, 1995, 1994). Esta carencia historiográfica ha sido también una constante en Europa hasta hace una década (Ruler y Verçic, 2004). Tales circunstancias son compartidas, además, por otras áreas geográficas, como América Latina y Asia (Culbertson y Chen, 1996; Molleda, 2000; Montenegro, 2004; Simoes, 1992; Sriramesh y Verçic, 2003; Wilson y Alozie, 2004). En general, los estudios históricos más relevantes provienen de los Estados Unidos. En consecuencia, en el cuerpo académico prevalece el modelo norteamericano para explicar el nacimiento y desarrollo de las relaciones públicas en otros países, sin hacer distinción entre situaciones políticas, sociales y económicas.

Desde los Estados Unidos se sitúa el origen de la disciplina en un ambiente democrático y de economía de mercado a principios del siglo XX. En la España de finales de los años 50, cuando encontramos los comienzos de la actividad, las condiciones políticas distaban de las americanas. La dictadura de Franco había comenzado en 1939 tras la Guerra Civil. Dicha situación política se prolongaría hasta la muerte del dictador en 1975, fecha en la que se abrirá un proceso democrático que instaurará una monarquía parlamentaria. Respecto a la existencia de una economía de mercado en nuestro país, resulta un presupuesto más discutible. A finales de los años 50, España se encontraba a las puertas del primer Plan de Estabilización que pretendía el saneamiento de la situación financiera y la progresiva liberalización del comercio. También por aquel entonces se apreciaban movimientos dentro del Ministerio de Información para promover una tímida libertad de prensa que no tomaría forma hasta 1966.

La verdadera transformación política, social y económica llegaría a finales de los 70. El inicio democrático acarreó una serie de reformas políticas y económicas que derivaron en una dinamización de la economía y del sector empresarial. La liberalización de ciertos sectores, junto a la aparición de unas Administraciones Públicas democráticas, conllevó una progresiva modernización del país, dando lugar a la estructura política, económica y social que hoy se conoce. Dichas circunstancias explican, en gran parte, por qué una profesión como las relaciones públicas comenzó entonces a cobrar importancia en las organizaciones.

El objetivo del presente artículo es exponer la evolución de las relaciones públicas a lo largo de las últimas cinco décadas, así como ofrecer un análisis de los primeros pasos profesionales y una panorámica de la reciente evolución profesional, comprendiendo así algunos factores clave que han ido configurando una 
práctica con gran presencia en la sociedad actual. Si bien los estudios históricos y teóricos de la comunicación, en general, tienden a centrarse en la natural relevancia de los medios de comunicación (Schlesinger, 1990), parece oportuno considerar y reflexionar sobre cómo se va configurando en el tiempo una disciplina profesional a través de su creciente importancia económica, formación e investigación académica. Por este motivo, en las siguientes páginas se describe y analiza el acontecer histórico desde una doble perspectiva: la práctica profesional y los primeros pasos doctrinales.

\section{Primeras campañas y escritos sobre relaciones públicas: 1953-1960}

La llegada a España de la expresión relaciones públicas y sus planteamientos teóricos fueron anteriores a las primeras campañas, aunque no demasiado. La práctica de las denominadas "campañas de relaciones públicas” comenzó en España a partir de 1958. Fue entonces cuando Joaquín Maestre, un joven español de la agencia de publicidad barcelonesa Danis, de visita en Amberes para asistir a un congreso de publicidad, tuvo un encuentro fortuito con Lucien Matrat. Los planteamientos del francés Matrat, padre y filósofo de las relaciones públicas europeas, interesaron sobremanera a Maestre. Este pionero español se dio cuenta de que las hasta entonces conocidas como "campañas de prestigio", desarrolladas por su agencia de publicidad Danis -en Barcelona- desde 1955 para diversos clientes, encajaban a la perfección con lo que en el resto de Europa se denominaban acciones de "relaciones públicas".

Las “campañas de prestigio" habían comenzado a finales de 1955, cuando Joan Fontcuberta, fundador de la agencia Danis, ideó para una marca conocida de productos de limpieza unos anuncios que pretendían mejorar la sociedad española. Se trató de una iniciativa concebida como una campaña que intentaba mejorar la sociedad de la época y que otorgaba prestigio a la marca: "No pretendía la venta de un producto, sino que era un apunte de programa social que pretendía una mejora de nuestra relación humana y que repercutió en un ambiente de buena voluntad para el producto y la firma que lo fabricaba" (Maestre, 1966) ${ }^{1}$. Bajo el lema Seamos mejores, la campaña se desarrolló en Cataluña entre octubre de 1955 y enero de 1956. El éxito de la iniciativa, tanto para el cliente como para la agencia Danis, resultó contagioso. Otros clientes solicitaron una campaña similar.

Como se intuye, fue Joaquín Maestre quien primero aplicó la etiqueta de "relaciones públicas" en la práctica, fruto de su conversación casual con Lucien Matrat en Amberes. Y, en este sentido, Joaquín Maestre puede considerarse como el pionero de la práctica en España. Su interés por el campo de las relaciones pú-

MAESTRE, J. (1966): “Antecedentes y evolución de las R.P. en España”, ponencia presentada en el I Congreso Español de Relaciones Públicas, Barcelona, mayo 1966, p. 3, recogido en el archivo profesional de Joaquín Maestre Morata -ARCH. JMM-PROF-. 
blicas iría en aumento, hasta el punto de hacerle albergar la idea de establecer su propia agencia dedicada íntegramente a ofrecer servicios de relaciones públicas. Así, en noviembre de 1960, Joaquín Maestre Morata fundó en Barcelona la primera empresa española de relaciones públicas: la S.A.E. de RP (Sociedad Anónima Española de Relaciones Públicas).

Aunque próximas en el tiempo, la práctica y la teoría de las relaciones públicas discurrieron de forma paralela en estos primeros años. En el ámbito de la teoría y de los estudios de comunicación, el primer autor que hizo referencia al concepto de "public relations" fue Pedro Prat Gaballí en 1953. El maestro publicitario catalán, en un párrafo de su obra Publicidad Combativa, se aventuró a traducir el término anglosajón por "relaciones generales" y las presentó sucintamente como un fenómeno empleado: "para aprovechar toda clase de coyunturas que brindan las relaciones personales para convertirlas en fomento de simpatía y base de confianza y prestigio en relación con la empresa y sus productos" (Prat Gaballí, 1953: 194). Al mismo tiempo que reconocía su novedad en España y en casi toda Europa, hacía constar que en Estados Unidos: "las grandes compañías, además de sus departamentos de Publicidad y de Ventas, sostienen un bien dotado Departamento de Relaciones Generales (Public Relations), que tienen por misión fomentar la cordialidad de las relaciones humanas de la empresa, dentro de la misma y en el palenque de la opinión pública" (Prat Gaballí, 1953: 60-61).

Con algo más de detenimiento, y ya bajo la traducción "relaciones públicas”, fue Juan Beneyto² quien académicamente se ocupó de ellas poco tiempo después. En su libro de 1957 Mass Communications. Un panorama de los Medios de Información en la Sociedad Moderna, dedicó uno de sus capítulos a las "Relaciones Públicas" (Beneyto, 1957:211-216)³. En apenas seis páginas, Juan Beneyto sitúa a las Public Relations como "una versión actual de la propaganda, de la psicología publicitaria" (Beneyto, 1957: 211). En este escrito, el autor menciona su origen anglosajón y las considera como un medio de comunicación que contribuye a la mejora de las relaciones entre la empresa y el público. Según Beneyto, las relaciones públicas ayudan a interpretar las actitudes del público y a formular una conducta consecuente de la empresa. El autor infunde un marcado carácter sociológico a la disciplina, puesto que no sólo se ocupa de mejorar la opinión, sino de forjar la buena voluntad con el público y la búsqueda de los desajustes que se puedan dar

Catedrático de Historia del Derecho, Juan Beneyto constituyó una de las piezas claves en la ciencia y enseñanza de la información en la España franquista. Llegó a ocupar el cargo de Director General de Prensa (del 7 de marzo de 1957, al 24 de enero de 1958) y Decano de la Facultad de Ciencias de la Información de Madrid. De ambos cargos fue cesado por no compartir la política del gobierno franquista.

3 Hay que tener en cuenta que la publicación recopila y "reelabora" el material que conformó el seminario sobre los medios de información contemporáneos y que impartió en el Instituto Social León XIII en la primavera de 1954. 
entre los objetivos y las realidades. Inmediatamente, ese enlace sociológico le lleva a plantear a las relaciones públicas como sucesoras de la propaganda política, como agentes que interactúan entre la sociedad y los gobiernos a través de la información. Tal vez esta visión teórica de la profesión fuera fruto del contexto político español -el régimen dictatorial de Franco-. Sin embargo, la práctica de la profesión en semejante contexto pudo ayudar a las empresas a presentarse ante una sociedad cada vez más abierta. La mejora del contexto económico con los planes de Estabilización económica también influyó y aseguró los primeros clientes para la empresa de Joaquín Maestre.

\section{Los años 60: la década de los intentos asociativos}

Como hemos apuntado, la década se inaugura con el nacimiento de la primera empresa española de relaciones públicas y se va a caracterizar por la búsqueda de entendimiento entre las primeras asociaciones profesionales. El recorrido por el movimiento asociativo y el reconocimiento profesional en esta década se puede dividir en tres periodos. El primero abarca los años comprendidos entre 1961 y 1965, años en los que aparecen la Agrupación Técnica de Relaciones Públicas en Barcelona y otras dos asociaciones: la Agrupación Española de Relaciones Públicas (Barcelona) y el Centro Español de Relaciones Públicas (Madrid), respectivamente. El segundo intervalo comprende los años 1966-1968, trienio marcado por la búsqueda de reconocimiento profesional y en el que destacan dos hechos: la celebración del I Congreso Español de Relaciones Públicas y la Asamblea anual de la International Public Relations Association-IPRA- en Barcelona. El tercer y último periodo se inicia en el año 1969, cuando se identifican los primeros esfuerzos reales de la comunidad profesional por institucionalizar las relaciones públicas.

El primer intento asociativo se produjo en 1961. La Asociación Técnica de Relaciones Públicas (ATRP) se constituyó el 8 de marzo de 1961 en Madrid para defender los intereses de la profesión y divulgar sus contenidos (Noguero, 1995: 142). Sus actividades no fueron más allá de la mera convocatoria de algunas conferencias y de la coordinación de la representación de la profesión en actos sociales durante apenas un par de años.

En estos años de precariedad asociativa, Joaquín Maestre, como destacado empresario del sector, procuraba el contacto con otros profesionales extranjeros, especialmente en congresos. Por ello, no dejaba de asistir a los organizados por la IPRA, de la que se hizo miembro. No tardaría en conseguir que el Consejo de la asociación eligiera a España como sede para su asamblea anual. El español argumentó ante el Consejo que la elección de España proporcionaría un impulso adicional a la profesión en un país que aún no contaba con una asociación profesional nacional y ayudaría, además, a establecer un mejor concepto para la etiqueta 
"relaciones públicas" . La argumentación de Maestre convenció al Consejo, que hubo de rechazar las solicitudes alternativas de Helsinki, Nueva York y Oslo como sedes ${ }^{5}$.

La XII Asamblea Anual de la IPRA se celebraría en Barcelona en 1966. Parece que fue la propia IPRA quien aconsejó a Maestre la constitución de una asociación profesional que reuniera a todos los técnicos españoles antes de la Asamblea. El empeño de Maestre propició que el proyecto de promover un nuevo intento asociativo profesional únicamente tardara tres meses en convertirse en realidad. Tras mantener algunas conversaciones con personas interesadas y técnicos en relaciones públicas, se consiguió reunir en Barcelona a un grupo de "profesionales ilusionados y dedicados con afán al ejercicio de un medio de comunicación social completamente nuevo en nuestro país: las Relaciones Públicas”6. Aquellos profesionales se convirtieron en agosto de 1965 en los socios fundadores de la Agrupación Española de Relaciones Públicas (AERP). De esa primera reunión surgieron proyectos para el futuro, concretados en unos estatutos que fueron elevados a la autoridad competente para su aprobación oficial, que no llegaría hasta un año más tarde .

Paralelamente se creó en Madrid otra asociación. Bajo el nombre de Centro Español de Relaciones Públicas (CENERP), fue promovida por Rafael Ansón Oliart y, en poco tiempo, autorizada por la Administración franquista. Unos meses después, Joaquín Maestre y Rafael Ansón coincidieron en una visita al Salón de la Infancia de París ${ }^{8}$. Ambos acordaron la conveniencia de unir las dos asociaciones en el año entrante, bajo el nombre común de CENERP, acompañado del distintivo de Madrid o Barcelona, según el caso. Pese a algunas diferencias iniciales en los planteamientos, primaba el deseo de unión, tal y como queda reflejado en unas palabras de Maestre en diciembre de 1965: "Ambos hemos acordado que, mientras que el Centro Español se encargará del centro de España, por ejemplo, Madrid y sus alrededores, la Agrupación Española se encargará especialmente del Norte de España. Cuando las circunstancias lo requieran, no habrá inconveniente en trabajar de manera conjunta y amistosa”.

4 La persuasiva intervención de Maestre quedó reflejada en las actas de la Asamblea: "Mr. Maestre extended an invitation to hold the next Council Meeting and General Assembly in Barcelona. He said that by doing so IPRA would be giving its support to a country which does not yet have a national public relations association, and help establish better understanding of true public relations work in a situation where many people calling themselves public relations consultants were offering services when in fact they were not bona fide colleagues". Actas de las sesiones remitidas por la Secretaría de la IPRA, ARCH. JMM-PROF: IPRA/65, pp. 14-15.

5 Cfr. Actas de las sesiones remitidas por la Secretaría de la IPRA, ARCH. JMM-PROF: IPRA/65.

6 Dossier informativo sobre la Asociación Española de Relaciones Públicas, copia personal de Luis Viñas Bona, vocal y posterior secretario de la Agrupación.

7 El Ministerio de la Gobernación visó y aprobó los estatutos el 26 de septiembre de 1966, cfr. Borrador del "Informe sobre la situación actual de las relaciones públicas en Barcelona por S.A.E. de Relaciones Públicas”, 25 de septiembre de 1969, ARCH. JMM-PROF: ASAMBLEA/69.

8 Entrevista personal a Joaquín Maestre, 6 de septiembre de 1999, Caldetas (Barcelona).

9 Extracto de una carta remitida por Joaquín Maestre a Robert Bliss, entonces presidente de la IPRA, el 21 de diciembre de 1965, ARCH. JMM-PROF: CONGRESIPRA/65. 
En una coyuntura de despegue industrial tras la puesta en marcha del I Plan de Desarrollo, el periodo que abarca los años 1966-1968 imprimió a la evolución profesional de las relaciones públicas un carácter agridulce. De un lado, 1966, el mismo año que contempló la Ley de Prensa que suavizó la censura previa franquista, constituyó un año muy especial y fructífero para la profesión. A la perspectiva de la celebración de la Asamblea anual de la IPRA en Barcelona hubo que añadir la de la unión de las principales asociaciones profesionales de España y la celebración conjunta del I Congreso Español de Relaciones Públicas ${ }^{10}$. Ambos acontecimientos, Asamblea y Congreso, tendrían lugar en Barcelona a finales de mayo de 1966.

A finales de octubre de 1966, tuvo lugar una reunión conjunta de los miembros del CENERP-Madrid y Barcelona. De ahí nació una Comisión Técnica, con el objeto de desarrollar una serie de estudios e investigaciones "como presupuesto indispensable para la profesionalización de las relaciones públicas"11. Entre los cometidos de la Comisión Técnica destacaba el estudio, tanto de los programas de acreditación de los profesionales, como de los requisitos mínimos que debía reunir la enseñanza de las relaciones públicas.

Junto a la creación de la Comisión, se estudió la posibilidad de organizar el II Congreso Español de Relaciones Públicas en junio de 1967. Sin embargo, la falta de empuje y coordinación impidieron su celebración. La aparente sintonía entre los capítulos de Barcelona y Madrid pronto quedaría en entredicho y cada uno continuaría su camino. Uno de los últimos logros que contempló el Centro Español como asociación nacional fue la incorporación de las relaciones públicas al Plan de Estudios de la Escuela Oficial de Periodismo ${ }^{12}$. Ante el nuevo fracaso asociativo, resurgiría poco después en Barcelona la Agrupación Española de Relaciones Públicas, decidida a impulsar el proyecto educativo de la constitución de una Escuela Superior de Relaciones Públicas. La ESERP puso en marcha su primer curso en febrero de 1968.

\section{Los años 70: en busca de la institucionalización de la profesión}

Este último periodo para la profesión antes de la democracia coincidió con las etapas de aplicación del II y III Plan de Desarrollo. Los objetivos económicos incluían la mejora de la estructura de los procesos pro-

10 Resulta evidente que la coincidencia de fechas no fue casual. Estuvo motivada por Maestre, quien a comienzos del año 66 manifestó: "Deberíamos aprovechar esta oportunidad para poder celebrar una reunión nacional de expertos en relaciones públicas, coincidiendo con las fechas inmediatamente posteriores a la Asamblea Internacional de la IPRA. Así, tendríamos la ocasión de ponernos en contacto con los hombres que desde hace años están intentado introducir bajo las coordenadas de verdad y honradez esta moderna y a la vez antigua técnica de las relaciones públicas". Nota redactada en papelería institucional de la IPRA y firmada por Joaquín Maestre, ARCH. JMM-PROF: CONGRESIPRA/66.

11 “Información del CENERP Barcelona a todos sus socios", documento en ARCH. JMM-PROF: CENERP/66.

12 Cfr. Orden Ministerial de 20 abril de 1967, publicada en el B.O.E. del 4 de mayo del mismo año. 
ductivos para aumentar la competitividad española, mantener la estabilidad interna y externa y asegurar el mantenimiento del pleno empleo. En el terreno político, en octubre de 1969, se produjo un cambio de gabinete que, entre otros, afectó al Ministerio de Información y Turismo. Alfredo Sánchez Bella sustituyó en el cargo de ministro a Manuel Fraga Iribarne en un momento crucial del proceso de institucionalización de las relaciones públicas. En estos años de camino hacia el reconocimiento legal de la profesión, destacan dos hechos: la I Asamblea Nacional de Relaciones Públicas, que intentará unir a todos los profesionales, y la creación del Registro Oficial de Técnicos en Relaciones Públicas.

El Centro Español de Relaciones Públicas (CENERP) de Madrid organizó la I Asamblea Nacional de Relaciones Públicas. En un gesto de acercamiento hacia la asociación de Barcelona, convocó oficialmente a los miembros de la Agrupación Española de Relaciones Públicas (AERP) a asistir y participar en ella. Se trataba de una nueva oportunidad para propiciar la unión y colaboración entre los profesionales españoles de las relaciones públicas. En resumen, se presentaba como "la definitiva Asamblea constituyente de las relaciones públicas en España"13 que, a su vez, abría la puerta a una futura institucionalización de la disciplina. La Asamblea se celebró los días 15 y 16 de octubre de 1969 en Madrid, bajo el patrocinio del Ministerio de Información y Turismo. El evento sirvió para establecer cauces de diálogo entre la profesión y la Administración (Tomás Bravo, 1981). Los participantes hicieron un verdadero esfuerzo por aunar intereses y elaborar conjuntamente un anteproyecto de ley para conseguir la institucionalización de las relaciones públicas. De este modo, se elaboró un informe que acordaba, entre otras cosas, la creación de una Comisión permanente que trabajara para lograr que la Administración reconociera oficialmente las relaciones públicas.

Dicha Comisión Permanente de la Asamblea Nacional de Relaciones Públicas no se constituyó hasta finales de noviembre, a la espera de los cambios ocurridos en el Ministerio de Información y Turismo, con el que, en última instancia, la Asamblea había acordado tratar los temas relativos a la institucionalización y profesionalización. El comienzo fue prometedor. El 14 de enero de 1970, una representación de la Comisión Permanente se entrevistó con el nuevo Subsecretario de Información y Turismo. La respuesta del Ministerio fue de comprensión e interés hacia la profesión y solicitó la elaboración -en un plazo de tres meses- de un informe que sirviera como base a la promulgación de un Estatuto legal de la actividad de relaciones públicas en España. Bajo el título: “Ordenamiento jurídico de las Relaciones Públicas”, el informe se elaboró en marzo de 1970 y supuso el paso previo a la elaboración del anteproyecto de Ley de Relaciones Públicas. En la reunión, el Ministerio reiteró el deseo de dotar a las relaciones públicas del oportuno ordenamiento

13 Así se expresaba en la carta circular que, con carácter urgente, la AERP dirigió a sus socios el 8 de octubre de 1969 para animarles a asistir, ARCH. JMM-PROF: AERP/69. 
jurídico. Se decidió crear una Comisión Mixta integrada por miembros del Ministerio y de la Comisión Permanente con el fin de elaborar conjuntamente el anteproyecto de disposición legal a la que, en principio, se otorgaría rango de ley. Finalmente, el anteproyecto fue presentado por el Ministro de Información y Turismo ante las Cortes en abril de 1971. Sin embargo, no sería finalmente aceptado por los ministros, ya que no se consideró que estuviera lo suficientemente "maduro" para ser tramitado por las Cortes.

A pesar de esta decepción cercana, el régimen franquista se despidió con algunos logros parciales para la profesión. En 1973, se creó la Agrupación Sindical Nacional de Técnicos en Relaciones Públicas, dentro del Sindicato Nacional de Prensa, Radio, Televisión y Publicidad. Dicha creación suponía cierto reconocimiento oficial, ya que cualquier entidad que deseara ser reconocida como asociación debía incorporarse antes a la Organización Sindical. El CENERP de Madrid había comenzado los trámites. Mientras, la AERP de Barcelona, de manera contraria a su proceder de los últimos años, se había desentendido de cualquier trámite en este sentido. El desinterés por conseguir el reconocimiento de la Administración vino motivado por un cambio en su Consejo Rector y por desavenencias internas. La etapa del nuevo Consejo Rector, encabezado por Federico Roda, coincidiría con un enfriamiento de las relaciones con los miembros de la Asociación Nacional Sindical de Técnicos en Relaciones Públicas-CENERP de Madrid.

Otros logros, aunque menores, de 1973, incluyeron la edición del citado Informe de la Comisión Permanente de la I Asamblea Nacional de Relaciones Públicas, patrocinada por cuatro de las empresas de relaciones públicas más emblemáticas: Augusto Ferrer, Célula, Jesús Ulled \& Asociados y S.A.E. de Relaciones Públicas. El 24 y 25 de mayo de 1973, además, tuvo lugar en Madrid la II Asamblea Nacional de Relaciones Públicas, convocada por la Agrupación Sindical de Técnicos en Relaciones Públicas de Madrid, en la que también participaron miembros de la AERP. En las conclusiones generales, se ratificó la necesidad de institucionalizar legalmente la actividad profesional. Se recomendaba aspirar a la aprobación de alguna norma legal con rango de Decreto a propuesta del Ministerio de Información y Turismo.

En esta línea de reconocimiento legal efectivo de la disciplina, el franquismo contempló dos últimos hechos dignos de mención. En primer lugar, la promulgación de una Orden Ministerial, el 14 de septiembre de 1974, por la que se aprobaba, aunque provisionalmente, el plan de estudios de la Facultad de Ciencias de la Información, que incluía la Licenciatura de Publicidad y Relaciones Públicas, como ampliación de la anterior Licenciatura de Publicidad. El plan de estudios provisional para todas las Facultades de Ciencias de la Información contemplaba la existencia de tres secciones en el segundo ciclo: periodismo, ciencias de la imagen visual y auditiva, y publicidad y relaciones públicas. No entrarían en vigor hasta 1992. En segundo lugar, dos nuevas Órdenes consecutivas, del 17 y 24 de abril de 1975, pasaron a regular la inscripción y cre- 
ación -respectivamente- del Registro Oficial de Técnicos en Relaciones Públicas del Ministerio de Información y Turismo (Noguero, 1994, 80).

De este modo, el régimen franquista, a través de la regulación, reconocía la importancia de las relaciones públicas y se despedía con una consideración casi profesional de una disciplina a la que contemplaba como una manifestación de la comunicación social. En el fondo, el Registro compensaba los esfuerzos llevados a cabo por el irregular movimiento asociativo que lograba, al menos, poder acreditar a todos los que ejercieran las relaciones públicas de forma profesional.

\section{1975: desarrollo profesional en democracia}

Ofrecer con cierta perspectiva histórica el desarrollo de una profesión durante las últimas tres décadas se antoja una labor compleja. Las páginas previas presentan un marco histórico sobre el acontecer profesional que goza de mayor perspectiva. Tal y como se ha analizado, en España, el nacimiento de las relaciones públicas se produjo en un contexto histórico particular. Las primeras "campañas de prestigio" de los años cincuenta cobraron forma de modo regular con actividades profesionales promovidas por empresas especializadas de relaciones públicas, como S.A.E. de RP, así como el surgimiento, en la década de 1960, de un animado debate sobre la naturaleza de la profesión y su incardinación en la estructura normativa y de reconocimiento legal. Merece la pena, ahora, adentrarse en la evolución de la disciplina desde entonces, insistiendo en que la descripción de las últimas tres décadas sólo ofrece una particular revisión de los principales acontecimientos. Dichos acontecimientos ofrecen ciertas conclusiones que sólo las décadas venideras podrán refrendar.

El advenimiento de la democracia en España a partir de 1975 motivó muchos cambios en los ámbitos político, social y económico. En este sentido, supuso un punto de inflexión para el mercado de la comunicación, que fue testigo de un crecimiento espectacular en la oferta y demanda informativas. El incremento del número de medios de comunicación, gracias a la consecución de la libertad de prensa (Barrera, 1995), constituyó uno de los acicates para que instituciones, tanto públicas como privadas, crearan sus primeros departamentos de comunicación.

Otra circunstancia, común a otros países, explica por qué empresas e instituciones crearon sus estructuras comunicativas: la progresiva consolidación de un régimen de libre mercado y, por tanto, de mayor oferta y necesaria diferenciación empresarial (Sotelo, 2004: 7). Hasta finales de los años ochenta, importantes sectores económico-empresariales -telecomunicaciones, energía, transportes, etc.- eran monopolios del Estado. Además, en la oferta de determinados productos y servicios existía un marco legal que hacía que al- 
gunos sectores -especialmente el financiero- ejerciesen su actividad bajo una marcada regulación. Sin embargo, el marco legal y económico en las últimas décadas del siglo XX se modificó sustancialmente, situando a las empresas ante un nuevo escenario económico y social.

Quizá uno de los aspectos más relevantes constituyó la consecución de la libertad informativa, con las primeras elecciones legislativas del 15 de junio de 1977. Éste fue uno de los motores que, unido a la liberalización económica, puso a empresas e instituciones ante la tesitura de responder a un entorno muy diferente, más dinámico y de complejas relaciones comunicativas. En este sentido, el ámbito empresarial es el que ha gozado de mayor vitalidad a la hora de organizar sus funciones comunicativas. Ante una mayor oferta de productos y servicios, las empresas deben ofrecer un mensaje corporativo que las diferencie en el mercado y, sobre todo, que transmitan de forma unívoca su identidad corporativa. Según Antonio López, el que fuera presidente de la Dircom -Asociación de Directivos de Comunicación de España-, la creación de departamentos de comunicación en las empresas se debió a "las exigencias del entorno. Luego surgieron en la prensa unos periodistas, que fueron los que dieron vida a la Asociación de Periodistas de Información Económica"14. Por su parte, instituciones públicas y sin ánimo de lucro progresivamente ven reforzado su papel en la esfera pública con una política comunicativa que responda al deber de informar (González, 2006; Canel, 2007).

Si bien en España existen escasas fuentes y referencias sobre el estado profesional durante aquellos primeros años de vida democrática (Moreno, 2004), las investigaciones promovidas por dos asociaciones profesionales relevantes (Dircom, 2000 y 2005; Adecec, 2002 y 2005) destacan dos fenómenos: la creación de departamentos de comunicación en instituciones españolas -públicas y privadas-a finales de la década de los setenta e inicios de los ochenta; y, en segundo lugar, el consiguiente crecimiento del sector de la consultoría que, según Teresa Dorn ${ }^{15}$, no se consolidaría hasta finales de la década de los ochenta.

14 Entrevista personal, Madrid, 31 de marzo, 2003. Antonio López, actual presidente de honor de la Asociación de Directivos de Comunicación, fue durante cuatro años (2000-2004) presidente de dicha asociación. Durante más de 25 años fue el máximo responsable de la dirección de comunicación del Banco Bilbao Vizcaya (1975-2001). Su dilatada trayectoria profesional le convierte en uno de los profesionales que iniciaron la dirección de comunicación como papel estratégico en las compañías.

15 Teresa Dorn fue una de las profesionales pioneras en el sector de agencias. En 1974 llegó a España para trabajar con Joaquín Maestre y abrir una delegación de S.A.E. de RP en Madrid. En 1981, contratada ya por Burson Marsteller, fue la encargada de abrir la primera oficina de la multinacional en Madrid. Formó parte del primer consejo rector de Adecec -junto con Jesús Ulled, y bajo la presidencia de Joaquín Maestre-, cuando fue fundada en 1991. Entrevista personal, 20 octubre de 2006, Pamplona. 


\section{Años 90: reconocimiento de la comunicación como activo estratégico. Nueva ola asociativa}

En la década de 1980 la profesión en España vive su primer boom. El segundo corresponderá a la siguiente década. El devenir de las circunstancias históricas políticas y económicas tuvo como punto de inflexión dos fechas citadas -1975 y 1977-, que supusieron la puesta en marcha de políticas económicas y modernización de la gestión pública. Los servicios comunicativos eran cada vez más demandados. Al crecimiento económico le acompañaría una inversión publicitaria progresiva (Etayo, 2002) a la que, paulatinamente, se le sumarían inversiones en otras áreas de comunicación no comercial. Instituciones y empresas requerían de la comunicación, que podía lograrse, bien por la contratación de agencias o consultoras, bien por la creación de estructuras internas con profesionales cualificados. La demanda de este tipo de servicios explica la apertura, durante los años ochenta, de las primeras oficinas de agencias internacionales de relaciones públicas ${ }^{16}$.

En este contexto, las grandes empresas, especialmente en el curso de las dos últimas décadas, han impulsado en su estructura interna unidades dedicadas a la gestión comunicativa (Dircom, 2000 y 2005). Su aparición podría ser definida como un cambio organizativo más en el natural desarrollo de las organizaciones. Sin embargo, una particularidad en el mundo empresarial reside en que la función comunicativa tiende a considerarse una herramienta estratégica situada en la alta dirección empresarial. Los directores de comunicación han escalado posiciones hacia la alta dirección, formando parte de la coalición dominante (Grunig, Grunig y Dozier, 2002: 65). Pese a que la actividad comunicativa ha sido siempre un elemento relevante, en el contexto español, un enfoque hacia una gestión estratégica e integral de la comunicación no comienza a ser un tópico habitual de discusión en foros profesionales hasta la última década del siglo XX, al igual que sucede en el entorno europeo (Cornelissen, 2004: 21; Ruler y de Lange, 2003: 156).

El desarrollo profesional de la última década del pasado siglo parece ir acompañado de una serie de circunstancias sociales y, fundamentalmente, económicas ${ }^{17}$. El crecimiento de la economía española y su

16 Como se ha indicado, la fundación, en 1960, de la primera agencia de relaciones públicas en España, S.A.E. de Relaciones Públicas, fue seguida por otras a lo largo de las dos siguientes décadas. No fue hasta entrada la democracia cuando las primeras agencias internacionales abrieron sucursales en Madrid. Las primeras agencias internacionales en hacerlo fueron Burson Marsteller y Hill \& Knowlton, ambas en Madrid en 1981. Otras le seguirían, bien con sus propias oficinas, o bien mediante adquisiciones de agencias nacionales: Ogilvy abrió oficina en 1991, Edelman en 1995. Otras agencias multinacionales llegaron a España a través de la adquisición de consultoras españolas. Así, la SAE de RP fue, por ejemplo, adquirida por Weber Shandwick en 1990, Ketchum adquirió S.E.I.S. en 1992 y Porter Novelli hizo lo propio con Comunicación Empresarial en 2002.

17 Similares conclusiones se hallan en el estudio de Miller y Dinan sobre el crecimiento de las relaciones públicas en Gran Bretaña durante las últimas décadas del siglo XX. Entre las circunstancias que destacan se encuentran la ola de privatizaciones empresariales emprendida por los gobiernos de Thatcher a principios de los ochenta. Hechos como éste motivaron, en opinión de los autores, una gradual importancia de las relaciones públicas en un mercado más competitivo. Cfr. Miller y Dinan, 2000. 
presencia internacional como una de las diez potencias económicas mundiales (OECD, 2007) se aprecia por la expansión internacional de las empresas mediante inversiones estratégicas, especialmente en países de América Latina y Europa (Horcajo, 2004). Por su parte, los mercados financieros españoles, con escaso protagonismo hasta mediados de los ochenta, se han convertido en motor de inversiones y dinamismo económico, motivando la aparición del llamado capitalismo popular, gracias, en parte, a los procesos de privatización de empresas públicas.

Este breve repaso del marco general durante las últimas tres décadas ayuda a comprender por qué corrientes profesionales como la gestión de la reputación corporativa y la responsabilidad social merecen atención no sólo en círculos académicos, sino que también forman parte de un animado debate profesional ${ }^{18}$. Tal y como señala Antonio López, "la gestión de los intangibles se configura como una línea de actuación de los comunicadores (...) La gestión de la reputación es ya un hecho a destacar en esta historia breve de los comunicadores" (López, 2006: 175).

Por otro lado, los movimientos asociativos de los años sesenta no tuvieron continuidad con la llegada de la democracia. El asociacionismo en relaciones públicas no tomaría el testigo de los intentos de las décadas precedentes hasta los noventa y con nuevas etiquetas: Adecec-Asociación de Empresas Consultoras en Relaciones Públicas y Comunicación- y Dircom -Asociación de Directivos de Comunicación-. Adecec fue instituida en 1991 por un grupo de representantes de las principales consultoras de relaciones públicas ${ }^{19}$. Actualmente la componen 32 empresas y es la patronal del sector más importante en España. Según los datos de la asociación, las empresas integrantes dan empleo a más de 1.000 personas y cuentan, en conjunto, con una facturación superior a los 100 millones de euros ${ }^{20}$. Al igual que Dircom, la asociación orienta su misión a "dignificar la profesión de las personas que trabajan en el sector y contribuir al crecimiento de la práctica de las relaciones públicas en España"21.

18 La reciente puesta en marcha en España de foros y organizaciones del ámbito profesional es una muestra. Dos entidades españolas relevantes son: Foro de Reputación Corporativa y Foretica. El Foro de Reputación Corporativa se constituyó en 2002, impulsado por 11 grandes empresas, ocho de las cuales pertenecen al selectivo índice bursátil Ibex 35 -dato de abril de 2008-. Dicho foro es el representante en España del Reputation Institute. En el ámbito de la responsabilidad social, la organización más relevante es Foretica -formada por más de 60 empresas y entidades-, nacida en 1999. Cfr. [http://www.foretica.es/foretica/?accion=objetivos].

19 Su primer consejo rector lo constituyeron Joaquín Maestre, Teresa Dorn y Jesús Ulled -fundador en 1965 de Ulled y Asociados Consejeros de Relaciones Públicas, segunda empresa española de relaciones públicas.

20 Adecec forma parte de la International Communication Consultancy Organisation-ICCO-.

21 Entre sus objetivos específicos se encuentran: "Impulsar y definir la práctica de la consultoría de comunicación y relaciones públicas (...) Fomentar y garantizar un alto nivel de calidad, profesionalidad y rigor ético de la profesión. Actuar de portavoz del sector ante las instituciones pública, empresas, otras asociaciones, líderes de opinión”. [http://www.adecec.com]. 
Por su parte, Dircom fue fundada en 1993 y, desde sus inicios ${ }^{22}$, uno de los propósitos fundamentales de la asociación se centró en potenciar en el ámbito profesional la consolidación de la comunicación “como herramienta estratégica para gestión de las organizaciones" ${ }^{23}$. Es interesante observar que la denominación elegida por la naciente asociación fuera dirección de comunicación, abandonando la terminología relaciones públicas que se adoptó en las primeras décadas de su nacimiento en España. Tal y como se apunta en un estudio de Adecec, "cuando preguntamos por la actividad propia de una agencia de relaciones públicas el desacuerdo es amplio. A ello contribuye quizá que, en los últimos tiempos, el término 'relaciones públicas' tiene en nuestra sociedad un componente peyorativo que ha provocado su caída en desuso por parte incluso de aquellos que principalmente se dedican a esta actividad" (Adecec, 2002: 25).

El activo papel de ambas asociaciones reafirma el constante crecimiento de este sector profesional, así como el interés permanente por lograr una ubicación relevante en las organizaciones. El empeño de los profesionales por obtener un reconocimiento del valor social de la profesión continúa siendo, igualmente en el ámbito internacional, una constante (Pieczka y L’Etang, 2001; Boynton, 2002).

Las investigaciones más ambiciosas para conocer el estado de la profesión en España no llegaron hasta el siglo XXI. Y dichos estudios vinieron de la mano de sendas asociaciones profesionales. Dircom publicó en el año 2000 El estado de la comunicación en España: empresas y organismos públicos e instituciones. Dicha investigación fue replicada en un segundo estudio que vio la luz en 2005 (Dircom, 2000 y 2005) 24

Las conclusiones que se derivan de estos estudios suponen una radiografía de cuál es el estado de la comunicación empresarial en España. Igualmente, son reflejo de las principales cuestiones que se discuten en el ámbito académico (De Santo y Moss, 2004; Ruler y de Lange, 2003; Grunig, Grunig y Dozier, 2002; Massie y Anderson, 2003).

22 Según la Memoria Anual 2006, la asociación comenzó en 1993 con 49 socios. En la actualidad, cuenta con más de 450. Dircom es socio fundador de la asociación internacional Global Alliance of Public Relations and Communication Management. Está además representada en el consejo europeo de la International Association of Business Communicators -IABC-. Cfr. [http://www.dircom.org]

23 Asimismo, la asociación centra sus objetivos específicos en: "Impulsar el reconocimiento profesional del director de Comunicación dentro de las organizaciones. Definir las competencias del director de Comunicación. Ser punto de referencia en la sociedad. Impulsar la formación permanente y la mejora profesional de los directivos de comunicación. (...)” Cfr. Dircom, en: [http://www.dircom.org]

24 Ambos estudios -2000 y 2005- sobre la comunicación en las empresas han tenido como muestra a responsables de comunicación de más de 200 empresas de mayor facturación en España de distintos sectores. Dircom publicó un estudio específico sobre la comunicación interna, Expectativas, prácticas y resultados de la comunicación interna en empresas e instituciones, Madrid, 2004. En 2008 se publicó un estudio dedicado a la comunicación en la Administración Pública, siendo una referencia más en el sector, dado que existen pocas investigaciones aplicadas en el sector público. 
En el sector de la consultoría, el crecimiento ha ido paralelo al de los departamentos internos de comunicación. Conforme los clientes han detectado necesidades comunicativas, además de crear estructuras comunicativas propias, también se han apoyado en los servicios de agencias y consultoras de comunicación. Los servicios de publicidad y comunicación comercial a lo largo del siglo XX en España han tenido una importancia creciente, sobre todo en cuanto a inversión publicitaria se refiere. Dichos servicios han sido apoyados y complementados desde la década de los ochenta por consultoras en comunicación y relaciones públicas. Desde que en 1982 se abriera la primera oficina de una agencia internacional -Burson Marsteller-, el sector ha experimentado un constante crecimiento, aunque el período de mayor crecimiento se situó entre los años 1996 y $2000^{25}$.

El crecimiento de la consultoría es relevante si tenemos en cuenta que se ha producido en un lapso de tiempo relativamente breve. Según los datos disponibles, la edad media de las consultoras no supera la década. Tal y como se ha descrito en las páginas iniciales, el nacimiento de la primera agencia de relaciones públicas hace casi 50 años fue, en su ámbito, pionera por la detección de necesidades entre los potenciales clientes. Sin embargo, no fue hasta la década de los noventa cuando el número de empresas se incrementó de manera notable. Tal y como afirma Juan Astorqui, quien fue consejero delegado de Burson Marsteller en España,

"La consultoría de comunicación ha evolucionado durante los últimos años desde lo que fue originalmente su actividad tradicional: la asesoría de relaciones públicas, que buscaba cómo asegurar que los clientes o sus productos disfrutaran de una notoriedad (...) Las nuevas áreas de actividad (...) reflejan la concienciación creciente entre las empresas y las asociaciones de intereses de que la comunicación constituye un elemento crítico para lograr sus objetivos básicos: la venta de productos y servicios, la formación de patrimonios de marcas..." (Astorqui, 1996: 122-123).

En definitiva, la consultoría en comunicación comparte con las empresas la tendencia hacia la profesionalización y el crecimiento futuro. Entre los factores que destacan los profesionales -consultoría y departamentos internos-, se encuentran la preocupación por la imagen corporativa, la obtención de credibilidad, la saturación de los medios tradicionales y la especialización por sectores.

La profesión se configura en el mercado español de manera similar a como lo hacen otros mercados de nuestro entorno. Las características sobre el contexto económico y social señaladas son un motor induda-

25 Según los datos de Adecec, el mayor crecimiento se experimentó en los años noventa, cuando el sector pasó de una facturación total media de más de 69 millones de euros en 1996 a algo más de 150 millones de euros en 2000. Cfr. Adecec (2002). La comunicación y las relaciones públicas en España. Radiografía de un sector. Madrid: Pirámide, p. 54. 
ble que permiten justificar y afirmar que la comunicación se está convirtiendo, progresivamente, en una herramienta de gestión. En este sentido, la situación española en la gestión comunicativa de las empresas, por lo general, sigue la pauta de otros países de nuestro entorno (Cornelissen y Thorpe, 2001; Fleisher, 1998).

\section{Conclusiones}

El nacimiento de la disciplina en España se produjo primero en la práctica profesional. Un grupo de profesionales del ámbito publicitario detectó con perspicacia las nuevas necesidades comunicativas de la sociedad de los años cincuenta. Según los hechos históricos descritos, la profesión moderna de las llamadas entonces relaciones públicas comenzó en una agencia de publicidad, para luego extenderse rápidamente a labores que raramente sobrepasaron acciones de publicity y organización de eventos en las primeras y escasas empresas de relaciones públicas de la España del tardofranquismo. Profesionales como Maestre intuyeron esa necesidad y, anticipándose a la demanda del mercado, establecieron una red de contactos internacionales que les permitió impulsar la práctica, organizarse como profesión e intentar aunar intereses.

El caso español no escapa a lugares comunes en la práctica internacional. Tanto en el desarrollo histórico estadounidense como en otros ámbitos geográficos -América Latina o Asia-parece hallarse una constante: el contexto socio-político y económico incide en el modo en que se desarrolla la profesión. Sin embargo, éste introduce en el debate, básicamente, ciertos matices sobre la función social que desempeña la práctica, tanto en contextos no democráticos como en los democráticos avanzados o incipientes. Tal es el caso de España, país en el que la profesión nació ligada al ámbito empresarial, en un régimen autoritario y enmarcada por unas circunstancias de desarrollo económico y tímida, aunque progresiva, apertura socio-política.

Los pioneros profesionales se preocuparon en España de institucionalizar la práctica y, al igual que había sucedido en algunos países europeos, y a diferencia de lo ocurrido en Estados Unidos, los primeros intentos asociativos de la profesión no tardaron en aparecer. Resulta especialmente relevante el hecho de que este paralelismo con Europa acontezca en un país y en unos años en que la estructura social carecía de tradición corporativista. Tal vez esta carencia se debiera, en parte, a la influencia de la Guerra Civil y los años de dictadura, que no fomentaban precisamente asociaciones cívicas ajenas a la iniciativa franquista. Este hecho, no obstante, pudo propiciar el fracaso a medio-largo plazo de las primeras asociaciones entre los practicantes de relaciones públicas de las dos ciudades principales, Barcelona y Madrid.

Las primeras asociaciones profesionales intentaron lograr en la década de los setenta, al final de la dictadura franquista, el reconocimiento de las relaciones públicas por parte de la Administración Pública. El 
proceso para lograr la ansiada institucionalización de la profesión culminó sin éxito en el periodo pre-democrático. No sería hasta 1994 cuando se obtuvo un mínimo reconocimiento institucional con la entrada en vigor de la Licenciatura de Publicidad y Relaciones Públicas en las Facultades de Comunicación.

En cuanto a la evolución de las denominaciones que ha ido recibiendo la profesión, hay que subrayar que el nacimiento de la profesión comenzó bajo la etiqueta relaciones públicas. Sin embargo, dicha denominación no ha tenido continuidad en la profesión durante el período democrático. Concretamente, a lo largo de las últimas dos décadas, otros términos -como comunicación corporativa, en el ámbito de la dirección y la reputación empresariales- parecen ir imponiéndose a la denominación original.Varios hechos pueden explicar esta circunstancia.

a) En primer lugar, la denominación "relaciones públicas" se importó directamente como traducción literal del término anglosajón public relations. El casual encuentro de Maestre con Lucien Matrat, y el innegable impulso y labor divulgadora que éste y otros profesionales españoles dieron a la expresión relaciones públicas fueron, sin duda, hechos que contribuyeron a asentar dicha denominación entre los profesionales que ejercían dichas prácticas. La traducción literal parece un intento por engarzar la tímida práctica española con la, sin duda, más amplia tradición histórica práctica y doctrinal de otros países, donde ya se reconocía la profesión -Estados Unidos, Gran Bretaña, Francia y Alemania, fundamentalmente.

b) En segundo lugar, las circunstancias históricas debieron influir en gran medida en la evolución de la profesión. En este sentido, los fracasos de los intentos asociativos ante el cambio de régimen -de la dictadura franquista a la democracia-pudieron ser una concomitancia de hechos que dejaron al término relaciones públicas en el terreno académico -heredero, además, de la dilatada tradición literaria anglosajona.

c) En tercer lugar, los últimos años parecen mostrar que los profesionales no se encuentran cómodos con dicha expresión. Según la visión profesional promovida, se ha dado un salto hacia el término dirección de comunicación. Con él se da a entender que la profesión aúna las diversas disciplinas y prácticas, en pos de una gestión comunicativa integrada.

Como se ha observado, el devenir de las circunstancias históricas -fundamentalmente políticas, económicas y sociales- explica la evolución profesional de los últimos años. El contexto económico y social revela por qué las empresas son las instituciones que más se han preocupado de impulsar la gestión comunicativa. En relación con el punto anterior, se explica, pues, que relaciones públicas se haya quedado como una 
denominación que para los profesionales no abarca la visión integral que debe tener la gestión en las organizaciones. En este sentido, los estudios sobre el estado de la profesión apuntan una idea fundamental: el director de comunicación tiende a ser el estratega y responsable último de la política comunicativa, que debe aunar áreas, tácticas y herramientas diversas.

Las últimas tendencias profesionales, que ponen especial acento en la gestión de la reputación y la responsabilidad social, no son sino muestra de una visión de los profesionales, como así queda recogida en los diversos estudios: la comunicación ha de lograr una gestión del intangible más relevante: la reputación. Por consiguiente, se convierte en una herramienta estratégica de dirección que debe coordinar esfuerzos comunicativos de la institución como un todo, con la natural distinción en función del complejo entramado de relaciones que emprende con sus diversos públicos.

¿Cuál es el futuro de esta disciplina y profesión? Parece imprudente, sobre todo si analizamos hechos históricos, aventurar el futuro. La disciplina en España es muy joven y la breve evolución histórica apunta ciertas tendencias que, según parece, se consolidan. Cómo ayudará la academia en su labor investigadora es, todavía, una asignatura pendiente. La práctica ha ido por delante de la teoría, hecho natural si tenemos en cuenta que hablamos de una disciplina que ha nacido de una necesidad real de las instituciones. El gran reto al que se enfrenta la doctrina en España es, como ya sucede en el contexto internacional, aportar valor analítico que revierta en una formación sólida de los futuros profesionales. A ellos corresponde estar preparados y ser conscientes de una realidad profesional tremendamente dinámica.

\section{Referencias bibliografícas}

Adecec, (2005): La comunicación y las relaciones públicas. Radiografía del sector 2004. Las consultoras de comunicación. Las empresas. Madrid.

Adecec, Iese y Pricewaterhousecoopers, (2002): El sector de la comunicación y las agencias de relaciones públicas en España, Madrid: Pirámide.

Arceo Vacas, A. (2002): "Public Relations in Spain in 2002: Antecedents, present situation and future tendencies", European Public Relations News, vol. 1, no 3, pp. 46-58.

Arceo Vacas, A. (2004a): “Public Relations in Spain: an introduction”, Public Relations Review, vol. 30, nº 3, pp. $293-302$.

Arceo Vacas, A. (2004b): "Principales acontecimientos en la evolución de las relaciones públicas en España”, en Arceo Vacas, J. L. (coord.): Las relaciones públicas en España, Madrid: McGraw Hill, pp. 51-56.

Arceo Vacas, J. L. (2006): “La investigación de relaciones públicas en España”, Anàlisi vol. 34, pp. 111-124. 
Arceo Vacas, J. L. (coord.) (2004): Las Relaciones Públicas en España, Madrid: McGraw Hill.

Barrera, C. (1995): Sin mordaza. Veinte años de prensa en democracia, Madrid: Temas de Hoy.

Beneyto, J. (1957): "Mass Communications". Un panorama de los Medios de Información en la Sociedad Moderna. Madrid: Instituto de Estudios Europeos.

Boynton, L.A. (2002): "Professionalism and social responsibility: foundations of public relations ethics", Communication Yearbook, 26, Mahwah: Lawrence Elrbaum Associates, pp. 230-265.

Canel, M.J. (2007): Comunicación de las instituciones públicas, Madrid: Tecnos.

Checa, A. (2007): “Historia de las RRPP”, en Checa, A. Historia de la Publicidad, La Coruña: Netbiblo, pp. 209-220.

Cornelissen, J. (2004): Corporate Communications. Theory and practice, Thousand Oaks: Sage.

Cornelissen, J.; Thorpe, R. (2001): “The organisation of external communication disciplines in UK companies: a conceptual and empirical analysis of dimensions and determinants”, The Journal of Business Communications, vol. 38, no 4 , pp. 413-438.

Culbertson, H.M. ; Chen, N. (1996): International public relations. A comparative analysis, Mahwah: Lawrence Erlbaum Associates.

Desanto, B.; Moss, D. (2004): "Rediscovering what Public Relations Managers do: Rethinking the Measurement of Managerial Behaviour in the Public Relations context”, Journal of Communication Management, vol. 9, no 2, pp. 179-196.

Dircom, (2005): El estado de la comunicación en España. $2^{\circ}$ Estudio. Madrid.

Dircom, (2000): El estado de la comunicación en España. I. Empresas II. Organismos públicos e institucionales. Madrid.

Etayo, C. (2002): “Advertising in Spain”, en Kloss, I. (ed.). More advertising worldwide, Berlin: Springer, pp. 238-269.

Fleisher, C.S. (1998): “A benchmarked assessment of the strategic management of corporate communications", Journal of Marketing Communications, vol. 4, pp. 163-176.

Fontcuberta, J. (1998): Hora Cero, Barcelona: Thassàlia.

González, H. (2006): Estrategias de comunicación en las ONG de Desarrollo. Departamentos, funciones e impacto en los medios, Madrid: Cideal.

Grunig, L. A.; Grunig, J. E.; Dozier, D. (2002): Excellent Public Relations and Effective Organizations. A Study of Communication Management in Three Countries, Mahwah: Lawrence Erlbaum Associates.

Horcajo, X. (2004): Al otro lado del Atlántico. La aventura equinoccial de la empresa española en América Latina, Barcelona: Laia Libros.

La Porte, J. M. (2001): Entusiasmar a la propia institución. Gestión y comunicación interna en las organizaciones sin ánimo de lucro, Madrid: Ediciones Internacionales Universitarias. 
López, A. (2006): “Diez años del anuario: algunas reflexiones sobre la profesión de los comunicadores”, en Dircom, Anuario de la Comunicación. $10^{\circ}$ aniversario, Madrid, pp. 173-178.

Losada Díaz, J. C. (coord.) (2004): Gestión de la comunicación en las organizaciones. Comunicación interna, corporativa y de marketing, Barcelona: Ariel.

Massie, L.; Anderson, C. L. (2003) : “Integrating communications: is the idea achievable?”, Corporate Communications: An International Journal, vol. 8, nº 4, pp. 223-228.

Miller, D.; Dinan, W. (2000): “The rise of public relations industry, 1979-1998”, European Journal of Communication., vol. 15, no 1, pp. 5-35.

Molleda, J.C. (2000): “International paradigms: the Latin American school of public relations", Journalism Studies, vol. 2, no 4 , pp. 513-530.

Moreno, M. A. (2004): “Spain”, en Ruler, B. van; Verçic, D. (eds.). Public Relations and Communication Management in Europe. A Nation-by-Nation Introduction to Public Relations Theory and Practice, Berlin: Mouton de Gruyter, pp. 393-412.

Noguero i Grau, A. (2004): "Principales acontecimientos en la evolución de las relaciones públicas en España”, en Arceo, J. L. (coord.): Las relaciones públicas en España. Madrid: McGraw-Hill, pp. 337-359.

Noguero i Grau, A. (1995): Función Social de las Relaciones Públicas: Historia, Teoría y Marco Legal, Barcelona: Escuela Superior de Relaciones Públicas.

Noguero i Grau, A. (1994): “La historia de las relaciones públicas en España: 1954-1990”, Revista Universitaria de Publicidad y Relaciones Públicas, n.1 (2a época), Máster en Comunicación Corporativa y Publicitaria, Universidad Complutense de Madrid, pp. 67-90.

Organisation for Economic Co-operation and Development (2007): OECD Factbook: Economic, Environmental and Social Statistics.

Pieczka, M.; L'Etang, J. (2001): “Public relations and the question of professionalism”, en Heath, R. L. (ed.): Handbook of public relations, Thousand Oaks: Sage, pp. 223-236.

Prat Gaballí, P. (1953): Publicidad Combativa, Barcelona: Editorial Labor.

Ruler, B. van; Verçic, D. (2004): Public Relations and Communication Management in Europe. A Nation-by-Nation Introduction to Public Relations Theory and Practice, Berlin: Mouton de Gruyter.

Ruler, B. van; De Lange, R. (2003): "Barriers to communication management in the executive suite”, Public Relations Review, vol. 29, no 2, pp. 145-158.

Schlesinger, P. (1990): "Rethinking the sociology of journalism: source strategies and the limits of media-centrism", en Ferguson, M. (ed.): Public communication: the new imperatives. Future directions for media research, London: Sage, pp. 61-83. 
Sotelo, C. (2004): “Historia de la gestión de la comunicación en las organizaciones”, en Losada Díaz, J.C. (coord.): Gestión de la comunicación en las organizaciones, Barcelona: Ariel, pp. 35-56.

Sriramesh, K.; Verçic, D. (eds.) (2003): The global public relations handbook. Theory, research, and practice, Mahwah: Lawrence Erlbaum Associates.

Tilson, H. M.; Alozie, E.C. (eds.) (2004): Toward the common good. Perspectives in international public relations, Boston: Pearson.

Tomás Bravo, C. M. (1981): “Las Relaciones Públicas en España”, en Lesly, P. (ed.): Nuevo Manual de Relaciones Públicas, Tomo II. Barcelona: Editorial Martínez Roca, pp. 427-457.

\section{Nota bibliográfica adicional}

El Fondo 144 Joaquín Maestre Morata (Arch. Jmm), consultado para la elaboración de este artículo, se encuentra en el Archivo General de la Universidad de Navarra, en el Fondo de Historia de España. 
